\title{
A direct numerical simulation of cool-flame affected autoignition in diesel engine-relevant conditions
}

\section{Nominated colloquium: IC engine combustion Word count: 5778 (method 2)}

\section{Main text: 4227 words, References: 614 Figure 1: 138 words, Figure 2: 162 words, Figure 3: 173 words, Figure 4: 264 words, Figure 5: 77 words, Figure 6: 123 words \\ Print in colour: no Supplementary material available}

\begin{abstract}
Alex Krisman ${ }^{\mathrm{a}, \mathrm{b}, *}$, Evatt R. Hawkes ${ }^{\mathrm{b}, \mathrm{c}}$, Mohsen Talei ${ }^{\mathrm{d}}$, Ankit Bhagatwala ${ }^{\mathrm{a}}$, Jacqueline H. Chen ${ }^{\mathrm{a}}$
${ }^{a}$ Combustion Research Facility, Sandia National Laboratories, Livermore, CA 96551-0969, USA

${ }^{b}$ School of Mechanical and Manufacturing Engineering, The University of New South Wales, Sydney, NSW 2052, Australia

${ }^{c}$ School of Photovoltaic and Renewable Energy Engineering, The University of New South Wales, Sydney, NSW 2052, Australia

${ }^{d}$ Department of fluid mechanics, The University of Melbourne, Melbourne, NSW 2052, Australia
\end{abstract}

\begin{abstract}
In diesel engines, combustion is initiated by a two-staged autoignition that includes both low- and high-temperature chemistry. The location and timing of both stages of autoignition are important parameters that influence the development and stabilisation of the flame. In this study, a twodimensional direct numerical simulation (DNS) is conducted to provide a fully resolved description of ignition at diesel engine-relevant conditions. The DNS is performed at a pressure of 40 atmospheres and at an ambient temperature of $900 \mathrm{~K}$ using dimethyl ether (DME) as the fuel, with a 30 species reduced chemical mechanism. At these conditions, similar to diesel fuel, DME exhibits two-stage ignition. The focus of this study is on the behaviour of the low-temperature chemistry (LTC) and the way in which it influences the high-temperature ignition. The results show that the LTC develops as a "spotty" first-stage autoignition in lean regions which transitions to a diffusively supported cool-flame and then propagates up the local mixture fraction gradient towards richer regions. The cool-flame speed is much faster than can be attributed to spatial gradients in firststage ignition delay time in homogeneous reactors. The cool-flame causes a shortening of the second-stage ignition delay times compared to a homogeneous reactor and the shortening becomes
\end{abstract}

\footnotetext{
${ }^{*}$ Corresponding author

Email address: ankrism@sandia.gov (Alex Krisman)
} 
more pronounced at richer mixtures. Multiple high-temperature ignition kernels are observed over a range of rich mixtures that are much richer than the homogeneous most reactive mixture and most kernels form much earlier than suggested by the homogeneous ignition delay time of the corresponding local mixture. Overall, the results suggest that LTC can strongly influence both the timing and location in composition space of the high-temperature ignition.

Keywords:

autoignition, direct numerical simulation, cool-flame, low temperature chemistry, negative temperature coefficient 


\section{Introduction}

Diesel engines play a major roles in the stationary energy and transportation sectors. The technology is relatively mature and robust, however, a fine-grained understanding of the in-cylinder process, which is needed to make further advances, is currently lacking. Of particular importance are the processes of ignition and flame stabilisation, which can significantly impact both fuel economy and the formation of pollutants such as nitrogen oxides and soot.

Conceptual models of diesel combustion have been developed based on observations in optically accessible engines and chambers [1-3]. Measurements of chemiluminescence, soot luminosity, and planar laser-induced fluorescence have been used to identify regions of low-temperature chemistry (LTC) and high-temperature chemistry (HTC), and to infer the existence of combustion modes such as nonpremixed combustion, the first and second stages of autoignition, and rich or lean premixed combustion.

Musculus et al. [3] presented conceptual models for conventional and low-temperature diesel combustion. A key feature of these models was the prominence of LTC. For conventional diesel combustion, LTC is present during both the ignition and quasi-stable phases. For low-temperature diesel combustion, a quasi-stable flame does not exist and the LTC occupies a large portion of the cylinder and persists long after the high-temperature ignition develops. Experimental observations cannot resolve the smallest spatial and temporal scales, and the number and type of observables are limited.

While the conceptual models outline the overall ignition process and identify the presence of LTC, a fine-grained understanding of the LTC behaviour and its effect on high-temperature ignition is still lacking. Direct numerical simulation (DNS) can complement physical experiments by targeting idealised configurations that resolve all temporal and spatial scales. Due to the extreme computational cost of applying DNS to experimentally measured diesel spray flames, a number of simplifying assumptions are necessary. Prior DNS [4-14] have targeted diesel-relevant thermochemical conditions by neglecting: detailed chemistry [4-6], three-dimensionality [4, 6-8, 10, 12-14], the spatial development of the jet $[4,5,8,10,11]$, and none of these studies included realistically intense turbulence. Nonetheless, several insights have been obtained. 
Sreedhara and Lakshmisha [5] considered the ignition of normal heptane in three-dimensional (3D) isotropic turbulence using a global chemistry model. Low-temperature autoignition was not discussed. They observed that high-temperature autoignition occurred in rich mixtures with low scalar dissipation rates $(\chi)$. This was consistent with previous DNS at non-diesel-relevant conditions which identified that autoignition occurs near a most-reactive mixture fraction value $\xi_{M R}[15]$ conditioned upon $\chi[15,16]$.

Mukhopadhyay and Abraham conducted two-dimensional (2D) DNS of ignition for laminar [8] and turbulent [10] mixing layers at diesel relevant conditions. A two-stage autoignition process was observed such that the first stage of autoignition was not sensitive to $\chi$ but the second stage of autoignition was delayed for high scalar dissipation rates [8]. For the turbulent case, both stages of autoignition occurred first at regions with low $\chi[10]$.

A DNS of lifted, laminar flame stabilisation with a reduced dimethyl ether (DME) chemistry by the present authors [12] identified several novel combustion features. Edge-flame, or hybrid edgeflame/autoignition structures were observed at diesel-relevant conditions. These flames resemble classic tribrachial (triple) flames with leading rich and lean premixed branches converging to a trailing nonpremixed branch at a triple-point. However, additional branches upstream of the triplepoint were observed that are due to either the first (LTC) or second (HTC) stages of autoignition. In particular, for the case where the oxidiser temperature was $900 \mathrm{~K}$, a single upstream branch was observed due to LTC. This branch was initiated at a mixture fraction, $\xi_{M R, 1}$, corresponding to the shortest first-stage ignition delay time, $\tau_{1}$, as calculated in a homogeneous reactor. A transport budget analysis [17] of the LTC branch revealed a transition from a convection-reaction balance to a diffusion-reaction balance. This suggested that the LTC branch transitioned from an autoignition to a diffusive "cool-flame". The LTC branch merged with the main tribrachial flame further downstream, producing a four-branched or tetrabrachial flame that is stabilised primarily by edge-flame propagation into a partially reacted mixture, a result that is broadly consistent with conceptual models of diesel combustion [2, 3].

Deng et al. subsequently observed a similar edge-flame to autoignition transition for lifted, laminar DME flames with increasing oxidiser temperature [13] and also inlet velocity [14]. In those studies, chemical explosive mode analysis (CEMA) [18] was applied, which provided further 
evidence for the hybrid edge-flame/autoignition structure and stabilisation mechanism that can exist for lifted, laminar flames at diesel engine-relevant conditions.

While several DNS of autoignition at diesel engine-relevant conditions have been conducted $[5-9,11]$, analyses of the LTC behaviour and LTC/HTC interactions have been limited. Given the prominence of LTC observed in diesel engine-relevant experiments $[2,3]$ and recent DNS of lifted laminar flames [12-14], further investigation of ignition at these conditions is merited. The present DNS considers an igniting, 2D, pseudo turbulent mixing layer at thermochemical conditions identical to the $900 \mathrm{~K}$ case from the previous laminar study [12]. These conditions were selected to reproduce the two-stage ignition and negative temperature coefficient (NTC) regime of ignition delay times that exists at diesel conditions and to investigate the interesting autoignition to deflagration transition observed in the spatially developing case, in the context of ignition. The main objective is to analyse in detail the behaviour of the LTC in the presence of 2D pseudo turbulence and to observe how it may affect high-temperature ignition.

\section{Configuration}

The DNS is conducted using S3D [19], a reactive flow solver for the compressible Navier-Stokes, conservation of energy, and conservation of species mass fraction equations. Spatial derivatives are approximated with an eighth order central difference operator and time is advanced with a six-stage, fourth order Runge-Kutta method. The solution is filtered every 10 time steps with a tenth-order explicit filter.

The domain, sketched in Fig. 1, is $2 \mathrm{D}$ and $3.6 \mathrm{~mm}$ by $3.6 \mathrm{~mm}$ in size, consisting of a periodic direction, $x$, and a non-periodic direction with outflow boundary conditions, $y$, in which the composition varies across a mixing layer of initial thickness $\sigma=25 \mu \mathrm{m}$ from air at $900 \mathrm{~K}$ at the bottom boundary, to fuel at $400 \mathrm{~K}$ at the top boundary. The system has a pressure of 40 atmospheres. A 2D domain was used due to the unfeasibly high computational cost of a 3D one at diesel-relevant conditions $^{1}$. The air is composed of $21 \% \mathrm{O}_{2}$ and $79 \% \mathrm{~N}_{2}$ by volume, and the fuel is composed of $70 \%$ DME and $30 \% \mathrm{~N}_{2}$ by volume. DME was selected because: it reproduces the two-stage ignition and

\footnotetext{
${ }^{1}$ The high cost is driven by the need to resolve extremely thin flames that occur at high pressure.
} 
NTC regime observed in diesel combustion, it is a potentially renewable, low-sooting alternative to diesel fuel, and its chemistry can be modelled with a compact chemical mechanism. The same 30 species reduced DME chemical mechanism [20] is used from the previous lifted laminar DNS study $[12]$.

Non-reflecting outflow boundary conditions were imposed in the $y$ direction. The initial mixture fraction $\xi$ profile is described by the expression: $\xi(y)=\frac{1}{2}\left(1+\tanh \left(\frac{y-\frac{1}{2}\left(y_{\mathrm{MAX}}-y_{M I N}\right)}{\sigma}\right)\right)$. Initial fuel and oxidiser profiles are set according to the mixing line while all other species are initially set to zero. The temperature profile is determined by adiabatic mixing between the fuel and oxidiser streams.

A decaying Passot-Pouquet turbulence spectrum [21] is superimposed at the initial time. The prescribed turbulence has a Damkhöler number, Da, of 0.4, defined as Da $=\tau_{E} / \tau_{\left(\xi=\xi_{M R}\right)}$, where $\tau_{E}$ is the eddy time defined as $\tau_{E}=L_{t} / u^{\prime} . L_{t}$ is the initial integral length scale (set equal to 0.1 $\mathrm{mm}$ ), $u^{\prime}$ is the initial root mean squared (RMS) turbulent velocity fluctuations (set equal to 0.49 $\left.\mathrm{ms}^{-1}\right)$. By the end of the simulation, time $t=1.14 \mathrm{~ms}$, the turbulent fluctuations have decayed to approximately two thirds of their initial value. $\tau_{\left(\xi=\xi_{M R}\right)}$ is the ignition delay time of the most reactive $\xi$, as measured in a homogeneous reactor. This Da is selected to match the approximate Da at the location of ignition under representative conditions, as estimated from Reynolds-averaged NavierStokes (RANS) computations of a normal dodecane spray flame [22]. The turbulent Reynolds number, $\operatorname{Re}_{t}=u^{\prime} L_{t} / \nu$, is equal to 23.4. This value is maximised within the constraints of the domain size and the target Da. The turbulent fluctuations are tapered to zero for regions near the $y$ boundaries, for numerical stability. Non-dimensional parameters are defined as follows: $x^{*}=x / L_{t}$, $y^{*}=y / L_{t}$, and $t^{*}=t / \tau_{\left(\xi=\xi_{M R}\right)}$.

The DNS mesh is uniform in the $x$ direction and stretched in the $y$ direction, with a fine-mesh region with a width of $1.8 \mathrm{~mm}$, centred at $y=0$. Due to the high pressure conditions, a very fine mesh was required. The fine-mesh region has a resolution of $1 \mu \mathrm{m}$ in both directions and the solution is advanced with a time step of $1 \mathrm{~ns}$. This resolution corresponds to at least 12 points across the thinnest reacting layers. The computational cost of the simulation was approximately 200,000 core hours on Intel Ivy Bridge processors. 


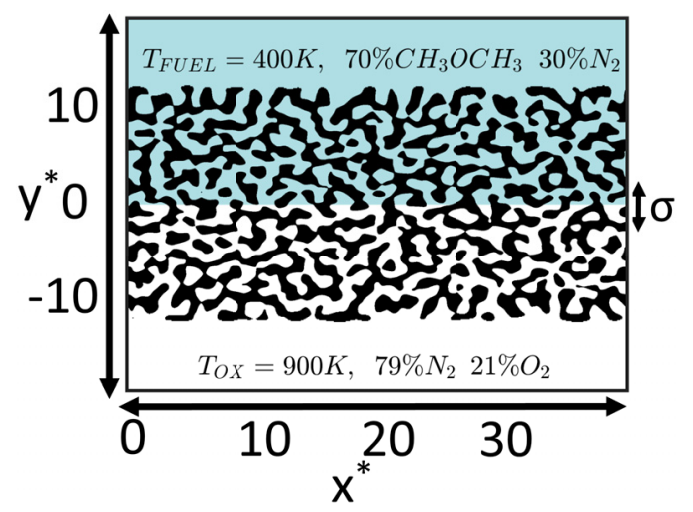

Figure 1: Initial domain configuration. Black shading pattern shows high vorticity regions. Grey/blue shading in top of figure represents the fuel and the white shading in the bottom represents the oxidiser.

\section{Results}

\subsection{Overview}

An overview of the evolution of the ignition is first presented for orientation. Figure 2 presents instantaneous images of heat release rate (HRR) for a fixed, subset of the domain (1.2 mm x 1.2 $\mathrm{mm}$ size). The stoichiometric mixture-fraction iso-surface is shown as a dashed white line. At $\mathrm{t}^{*}$ $=0.4 \mathrm{HRR}$ is observed in lean mixtures developing with a "spotty" pattern and a magnitude of order $1 \times 10^{10} \mathrm{~W} / \mathrm{m}^{3}$. At $\mathrm{t}^{*}=0.8$, the peak HRR values have increased to order $1 \times 10^{11} \mathrm{~W} / \mathrm{m}^{3}$ and have formed into a connected, wrinkled surface, moving into richer mixtures. Simultaneous visualisations of the LTC species methoxymethyl peroxide radical $\mathrm{Y}_{\mathrm{CH}_{3} \mathrm{OCH}_{2} \mathrm{O}_{2}}$ (not shown here) confirm that the HRR up to this point is due to LTC. Inspection of the unconditioned peak values of $\mathrm{Y}_{\mathrm{CH}_{3} \mathrm{OCH}_{2} \mathrm{O}_{2}}$ (not shown here) indicates that the first-stage of autoignition occurs at $\mathrm{t}^{*}=0.44$ $(\mathrm{t}=0.22 \mathrm{~ms})$ and $\xi=0.07^{2}$. These values are very lean, but less lean than the homogeneous reactor values of $\tau_{1}=0.19 \mathrm{~ms}$ and $\xi_{M R, 1}=0.03$ (as reported in Ref. [12]). Analysis presented in section 3.2 will show that the HRR at $\mathrm{t}^{*}=0.4$ corresponds to the first stage of autoignition and that the HRR at $\mathrm{t}^{*}=0.8$ corresponds to a cool-flame. A sudden increase in HRR develops at $\mathrm{t}^{*}=1.2$ at a point which is rich, but less rich than the region where HRR is occurring due to LTC. Simultaneous visualisations of the HTC species hydroxyl radical $\mathrm{Y}_{\mathrm{OH}}$ (not shown here) confirm that the intense point of HRR is an HTC ignition kernel (the second stage of autoignition)

\footnotetext{
${ }^{2}$ The first stage of autoignition is defined to be when the unconditioned maximum value of $\mathrm{Y}_{\mathrm{CH}_{3} \mathrm{OCH}_{2} \mathrm{O}_{2}}$ exceeds $20 \%$ of the maximum value observed over the simulation [12].
} 
which is forming about $20 \%$ later than expected from the homogeneous value, $\tau_{\left(\xi=\xi_{M R}\right)}$. The kernel rapidly expands and by $t^{*}=1.4$ it has engulfed the $\xi_{S T}$ surface and established two edge-flames, similar to the ignition dynamics previously observed in non-diesel-relevant conditions [23, 24]. The edge-flames have a main tribrachial (triple) flame composed of a crescent-shaped leading lean and rich premixed branches that merge at the triple-point with a trailing nonpremixed branch and this propagates into regions of LTC which can be considered a fourth branch of the edge-flames. This resembles the tetrabrachial (quadruple) flames observed at identical thermochemical conditions in a prior lifted, laminar flame study [12]. A second ignition kernel that formed at $t^{*}=1.3$ (not shown here), is rapidly expanding through rich mixtures at $t^{*}=1.4$ as a front of intense HRR. For the entire domain (not shown here), twenty ignition kernels are observed, staged from $\mathrm{t}^{*}=1.2$ to 2.0. Each kernel develops as a spatially distinct ignition which behaves similarly to the two kernels observed in Fig. 2, although some kernels merge with preexisting kernels or the rich premixed branches of established edge-flames before they can reach the $\xi_{S T}$ surface.

Overall, Fig. 2 shows that the first-stage of autoignition initiates at lean mixtures, but that LTC then moves quickly into richer mixtures. The second-stage of autoignition is observed at rich mixtures in spatially distributed kernels. The ignition kernels rapidly expand and establish tetrabrachial edge-flames which resemble stabilised laminar flames at identical thermochemical conditions [12]. However, the kernel expansion and edge-flame propagation is not the focus of this study. A detailed analysis of these features will be included in future work. The focus of the remainder of this paper is on the development of the LTC and its influence on the high-temperature ignition.
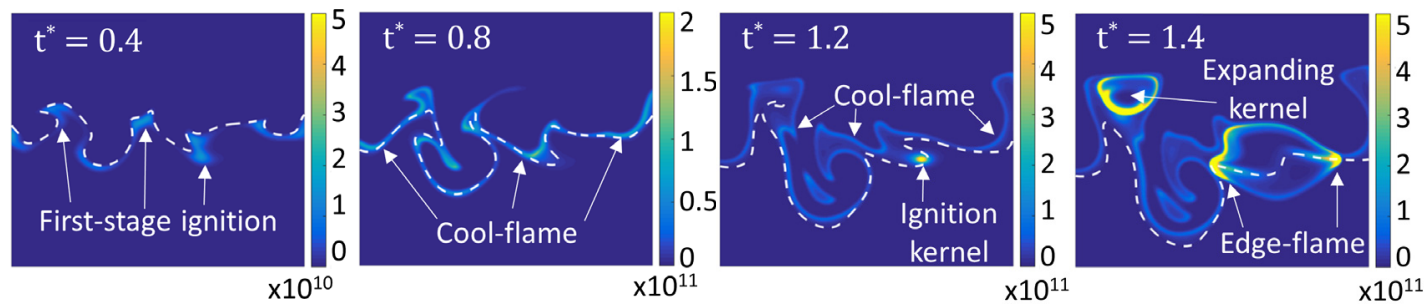

Figure 2: HRR for a fixed window of the domain. The lower portion of the each sub-figure corresponds to the lean side of the mixing layer. The dashed white line is the $\xi_{S T}$ surface. Note that the colour scale is not fixed. 


\subsection{Low temperature chemistry}

In this section, the LTC ignition is examined, with a particular focus on how it is affected by molecular diffusion. There are two kinds of diffusional influences that may be important. First, previous research for single stage autoignition has noted that ignition kernels are affected by diffusion, as they are observed to occur in regions close to $\xi_{M R}$ where the $\chi$ are the lowest $[4,6,7,15,25-31]$. Second, as reported in Ref. [12], there exists a gradient of homogeneous first-stage ignition delay times in $\xi$ space. Therefore, it may be argued that the observed movement of the LTC into richer $\xi$ is due to a gradient in $\tau_{1}$. This would be the low-temperature equivalent to a spontaneous ignition front that can occur for high-temperature ignition in stratified mixtures [32]. However, it is also possible that the LTC is a diffusively-supported "cool-flame" [33], that propagates with its own velocity which is different from the reciprocal of the gradient of $\tau_{1}$.

Examining the first kind of diffusion effect (the delayed ignition in discrete kernels due to high $\chi)$, it is noted that Fig. 2 shows that the first stage of autoignition develops from several isolated locations, indicating the existence of preferred locations for the first stage of autoignition. To see if this spottiness is explained by $\chi$, statistics of HRR doubly conditioned upon $\xi$ and $\chi$ are presented here from $\mathrm{t}^{*}=0.2$ to $\mathrm{t}^{*}=0.8$ (a period with negligible HTC) in Fig. 3. The results are first conditioned upon $\xi$, and then conditioned on ranges of $\chi$.

At $\mathrm{t}^{*}=0.2$, the HRR has a strong dependency on $\chi$, such that lower $\chi$ values are associated with higher HRR. This shows that the LTC is initiated preferentially in regions with low $\chi$, which is consistent with a prior DNS study of an autoigniting n-heptane mixing layer [10]. This observation also explains why the HRR develops in isolated spots rather than uniformly in $\xi$ space, since stronger dissipation is suppressing LTC. This behaviour continues to $t^{*}=0.4$. During this time the magnitude of HRR increases. This sensitivity of LTC to $\chi$ during ignition is analogous to the $\chi$ sensitivity observed for high-temperature autoignition of single-stage ignition fuels in stratified mixtures [4, 15, 27-31], where ignition first occurs at locations of minimum $\chi$, conditioned on a $\xi_{M R}$.

By $\mathrm{t}^{*}=0.6$, the HRR has increased to a quasi-steady value (until the HTC commences). The dependence of HRR on $\chi$ has now qualitatively changed such that the strongest HRR occurs for intermediate levels of $\chi\left(50<\chi<100 \mathrm{~s}^{-1}\right)$. That is, the monotonically decreasing dependence of 
HRR on $\chi$ has disappeared. Extreme levels of dissipation $\left(\chi>500 \mathrm{~s}^{-1}\right)$, still cause a reduction of HRR at most $\xi$ values.

From $\mathrm{t}^{*}=0.6$ to $\mathrm{t}^{*}=0.8$, the evolution of $\mathrm{HRR}$ with respect to $\xi$ is qualitatively similar for $0<\chi<500 \mathrm{~s}^{-1}$ (HRR remains suppressed for $\chi>500 \mathrm{~s}^{-1}$ ) and peak values of HRR move into richer mixtures. Since the LTC is moving into richer $\xi$-values, it is important to consider the rich side of the distributions which are promoted by moderate to high levels of $\chi$. This indicates that diffusive effects are promoting the movement of LTC into richer mixture fractions.

The role of diffusion in supporting the LTC can be quantified by considering the transport budget analysis [17] for the LTC marker $\mathrm{Y}_{\mathrm{CH}_{3} \mathrm{OCH}_{2} \mathrm{O}_{2}}$. Figure 4 presents reaction and diffusion terms of the transport budget of $\mathrm{Y}_{\mathrm{CH}_{3} \mathrm{OCH}_{2} \mathrm{O}_{2}}$ in addition to $\chi$, evaluated on lines aligned with the $\xi$ gradient. These terms are evaluated at an early time, $\mathrm{t}^{*}=0.4$, when the HRR is lower and the LTC is isolated in discrete regions, and a later time, $\mathrm{t}^{*}=0.8$, when the HRR is higher and the LTC is established as a wrinkled surface.

At $t^{*}=0.4$, four lines of evaluation were selected: $\mathbf{A}, \mathbf{B}, \mathbf{C}$, and $\mathbf{D}$. For all lines, the reaction magnitude is far larger than that of diffusion, confirming that the HRR peaks at $\mathbf{A}, \mathbf{B}$, and $\mathbf{C}$ are localised regions of first stage of autoignition. The differences in magnitude of HRR between the line segments correlates with the $\chi$ profiles. The lines with the location of intense HRR, A and $\mathbf{C}$, have the lowest $\chi$, the line $\mathbf{B}$ with intermediate HRR has higher $\chi$, and the line $\mathbf{D}$ with the strongest $\chi$ is not experiencing significant HRR. This relationship between $\chi$ and the formation of LTC is consistent with the results observed in Fig. 3.

At $\mathrm{t}^{*}=0.8$, transport budget terms are evaluated along the lines $\mathbf{E}, \mathbf{F}, \mathbf{G}$, and $\mathbf{H}$. For all lines, the reaction and diffusion terms have comparable magnitudes and opposite sign. This is characteristic of a diffusely-supported flame, or deflagration. For low to intermediate $\chi$, corresponding to lines $\mathbf{E}, \mathbf{F}$, and $\mathbf{H}$, the strength of the HRR is similar. Conversely, line segment $\mathbf{G}$ is experiencing very high $\chi$ and low HRR.

The transport budget analysis, in conjunction with the doubly conditioned results, supports the notion of the LTC transitioning from a series of localised autoignition events (located near $\xi_{M R, 1}$ and very low $\chi$ ) into a diffusely supported "cool-flame" that propagates into richer mixtures.

In order to identify the location of the cool-flame, a volume may be defined that bounds the 
HRR due to LTC. A definition was identified after considering several minor LTC species. The species $\mathrm{OCH}_{2} \mathrm{OCHO}$ is a short-lived intermediate species, associated purely with LTC, that has a peak value which is collocated with the maxima of HRR and is appropriate for defining the location of the cool-flame. Here, the cool-flame is defined to exist where $\mathrm{Y}_{\mathrm{OCH}_{2} \mathrm{OCHO}}>\mathrm{Y}_{\mathrm{OCH}_{2} \mathrm{OCHO}_{C R I T}}$, where $\mathrm{Y}_{\mathrm{OCH}_{2} \mathrm{OCHO}_{C R I T}}=2.21 \times 10^{-5}$, which is $20 \%$ of the maximum value observed during the simulation. Since $\mathrm{OCH}_{2} \mathrm{OCHO}$ is not produced by $\mathrm{HTC}$ reactions and is also consumed at high temperatures, it successfully isolates regions of LTC. For further details of this cool-flame definition, see the supplementary material.

Figure 5 presents the evolution of the PDF for $\xi$ conditioned upon the cool-flame locations. The PDF starts as a very narrow profile at lean $\xi$. As time progresses, the PDF moves into richer $\xi$ and becomes increasingly broad up until the point of high temperature ignition, which occurs at approximately $\mathrm{t}^{*}=1.4$. Following the high temperature ignition, the PDF continues to move into richer $\xi$, but the PDF becomes more narrow. This corresponds to the consumption of the cool-flame by the HTC, which occurs from less rich mixtures. By the end of the simulation the cool-flame is confined to extremely rich mixtures.

The LTC behaviour may be summarised as:

1. The first stage of autoignition develops at a composition of $\xi=0.07$ and a time of $\mathrm{t}^{*}=0.44$ $(\mathrm{t}=0.22 \mathrm{~ms})$. The equivalent values from simulations of a homogeneous reactor are $\xi_{M R, 1}=$ 0.03 and $\tau_{1}=0.19 \mathrm{~ms}$, respectively.

2. The first stage of autoignition is initiated at spatially isolated regions that have a similar $\xi$ value, and a low $\chi$ value. The LTC initially exhibits a sensitivity to $\chi$ similar to that observed for single-stage autoignition.

3. The LTC regions form into a connected, wrinkled surface that is supported by diffusion. This feature is termed a cool-flame.

4. The cool-flame moves up the mixture fraction gradient into richer mixtures. LTC reactivity at the leading (richer) side of the cool-flame is promoted by moderate to high levels of $\chi$.

5. Following the high temperature ignition, the cool-flame and the regions of LTC are gradually consumed from less-rich $\xi$, while the cool-flame continues to move up the $\xi$ gradient. 

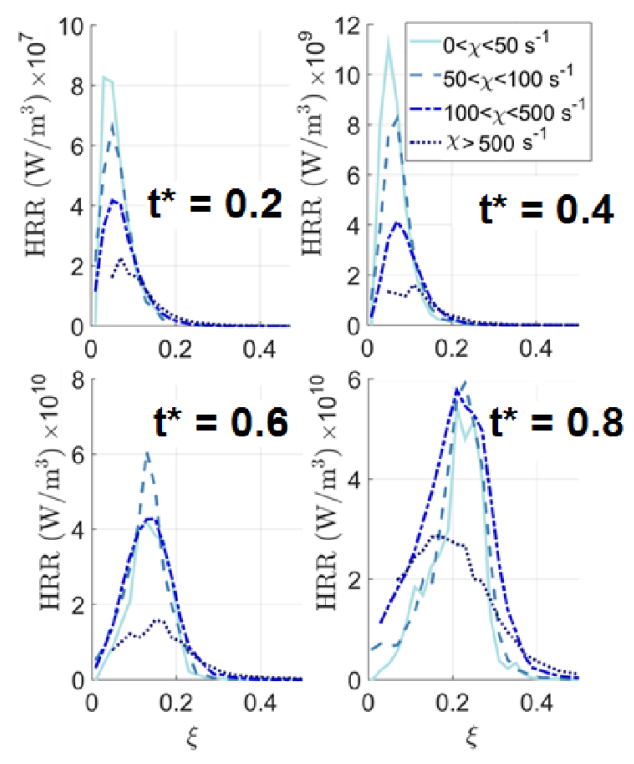

Figure 3: HRR means conditioned on $\xi$ and $\chi$.



Figure 4: Evaluation of transport budget for $\mathrm{Y}_{\mathrm{CH}_{3} \mathrm{OCH}_{2} \mathrm{O}_{2}}$. Top row shows the HRR field, superimposed with the lines used to evaluate the transport budgets. The lower sub figures show the diffusion (D) and reaction (R) terms on the left y-axes and $\chi$ on the right y-axes. Negative and positive values of distance correspond to the oxidiser and fuel side of the mixing layer, respectively. 


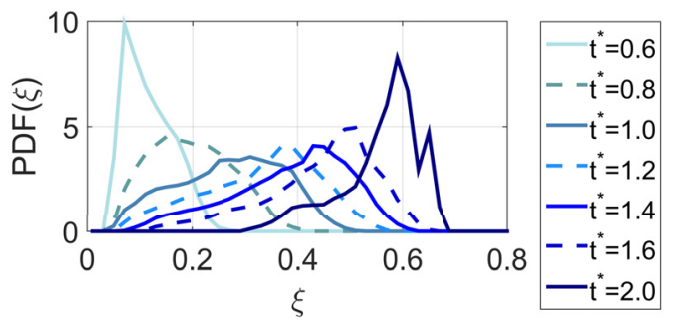

Figure 5: PDF conditioned upon cool-flame location, for $\xi$.

\subsection{High-temperature ignition}

An ignition kernel is defined as a local maxima of HRR which emerges from a point-source due to autoignition. The kernel formation is defined as the instant at which the peak in HRR exceeds $4 \times 10^{11}\left(\mathrm{~W} / \mathrm{m}^{3}\right)$. This threshold was selected in order to rule out the inclusion of any region of LTC.

As shown in Fig. 6, twenty kernels formed in rich $\xi$, much richer than expected from the homogeneous most reactive mixture fraction, $\xi_{M R}=0.11$. The first ignition kernel formed at $\xi=$ 0.25, and subsequent kernels form between 0.14 and 0.50 . The first ignition kernel occurred at a very low dissipation rate, $\chi=11\left(\mathrm{~s}^{-1}\right)$, but subsequent kernels form between 0 and $247\left(\mathrm{~s}^{-1}\right)$.

This result has implications for the conceptual models of diesel combustion. Since the $\xi$ distribution is closely related to mixing, the exact location of kernel formation may be important for the overall ignition and stabilisation process. A recent conceptual model for conventional diesel conditions [3] states that autoignition initiates from rich mixtures, and chemiluminescence measurements suggest that the ignition develops from an ensemble of distributed kernels [1]. However, it is not possible to simultaneously measure instantaneous ignition kernel markers and the local mixture composition [34]. Instead, the location of the ignition kernels in $\xi$ space may only be inferred in an approximate sense, e.g. see Refs. $[34,35]$. For this reason, it is difficult to determine from experimental results if the autoignition kernels are forming at $\xi_{M R}$, or some other value or range of values. The present results suggests that $\xi_{M R}$ may not be sufficient to predict the location of autoignition, and that the ignition kernels are able to form over a wide range of mixture fractions.

It has been observed in the current DNS that the formation of autoignition kernels in rich mixtures is preceded by the passage of a cool-flame. This raises the possibility that the cool-flame is affecting the behaviour of the high temperature ignition, and may explain why the $\xi_{M R}$ value 


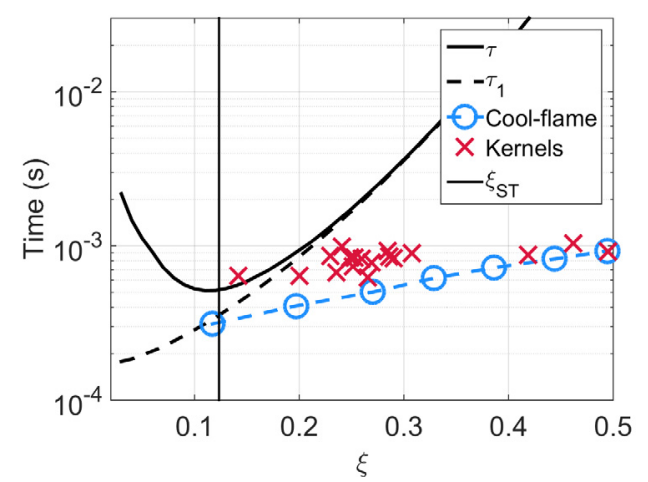

Figure 6: Comparison of $\tau_{1}$ and $\tau$ with the cool-flame and ignition kernels. The circle markers show the cool-flame. The cross markers show the locations of the ignition kernels.

does not predict the location in composition space where the ignition kernels form. To investigate the effect of the cool-flame on kernel formation, the movement of the cool-flame and the formation of the ignition kernels in $\xi$-space are compared to the first-stage and main homogeneous ignition delay times, $\tau_{1}$ and $\tau$ respectively, in Fig. 6. Here, the cool-flame location is defined by the mean $\xi$ value evaluated on the cool-flame.

The kernels are forming at times that are $20 \%$ to $100 \%$ longer than $\tau_{\left(\xi=\xi_{M R}\right)}$. However, when compared to the $\tau$ value at the corresponding $\xi$ value, all kernels except one are igniting much earlier than expected. The results also show that the cool-flame originates from lean $\xi$, initially near the $\tau_{1}$ curve. However, over time, the cool-flame progresses into richer $\xi$ much faster than $\tau_{1}$. The shortening of the first stage of autoignition by the cool-flame becomes more pronounced at richer $\xi$. Since the dwell between $\tau_{1}$ and $\tau$ times decreases with increasing $\xi$, the shortening of the overall ignition delay is expected to also become more pronounced with increasing $\xi$, which is indeed observed. Acceleration of autoignition due to diffusion and conduction from nearby HTC kernels is ruled out, since the kernels occur at spatially isolated locations

To the best of authors' knowledge, this property of NTC-affected ignition has not been previously identified. The introduction of a rich-bias to the location of kernel formation is broadly consistent with the limited available experimental data [1,3], and recent transported PDF modelling of ndodecane spray flames [22]. However, the current results do not consider the effect of intense turbulence that exist at diesel conditions, which may preclude the cool-flame influence on the kernel formation location. Further investigation at realistic turbulent conditions is required. 


\section{Conclusions}

A two-dimensional DNS of an igniting mixing layer subject to decaying isotropic turbulence was conducted. The selected thermochemical parameters were representative of diesel engine operating conditions. The results show that the ignition dynamics are complex and involve low and high temperature autoignition and the propagation of edge-flames that resemble the hybrid premixed/autoignitive tetrabrachial flames observed in identical thermochemical conditions in a prior lifted laminar flame study [12].

The initial turbulent spectrum was selected to approximate the Da value near the ignition location relevant to diesel combustion. This resulted in a mixing field that created isolated regions of low $\chi$ and reactive $\xi$ values to establish the first stage of autoignition. The timing and location of the first stage of autoignition corresponded to the value obtained from homogeneous reactor simulations. The first stage of autoignition rapidly evolved from discrete ignition kernels into a wrinkled front of LTC, that was identified as a diffusively-supported deflagration, or cool-flame. The cool-flame propagated up the $\xi$ gradient much faster than could be accounted for by gradients in the first stage ignition delay time. This resulted in the first-stage ignition delay time being shortened preferentially at richer mixture fractions. The autoignition kernels (second stage of autoignition) formed in mixtures much richer that expected from a homogeneous reactor, and this is concluded to be due to the increased reactivity induced by the cool-flame.

The identification of the cool-flame as a distinct combustion feature that significantly affects the HTC ignition is a key finding of this study. The effect of the cool-flame on the location and timing of the formation of autoignition kernels may have important ramifications for the modelling of combustion in diesel-like conditions and merits further investigation, particularly with regards to the effects of realistic, three-dimensional turbulence.

\section{Acknowledgements}

This work was supported by the Australian Research Council. The work at Sandia National Laboratories was supported by the Combustion Energy Frontier Research Center, an Energy Frontier Research Center funded by the US Department of Energy (DOE), Office of Science, Office of Basic Energy Sciences under Award No. DE-SC0001198. Sandia is a multiprogram laboratory operated 
by Sandia Corporation, a Lockheed Martin Company, for the United States Department of Energy

under contract DE-AC04-94AL85000. The research was supported by computational resources on the Australian NCI National Facility through the National Computational Merit Allocation Scheme and Intersect Australia partner share and by resources at the Pawsey Supercomputing Centre.

\section{References}

[1] J.E. Dec, SAE Paper 1997-97-0873, (1997).

[2] C.A. Idicheria, L.M. Pickett, SAE Paper 2006-01-3434, (2006).

[3] M.P.B. Musculus, P.C. Miles, L.M. Pickett, Prog. Energ. Combust. Sci. 39 (2013) 246 - 283.

[4] S. Sreedhara, K.N. Lakshmisha, Proc. Combust. Inst. 28 (2000) $25-33$.

[5] S. Sreedhara, K.N. Lakshmisha, Proc. Combust. Inst. 29 (2002) 2051 - 2059.

[6] A. Viggiano, Combust. Flame 137 (2004) 432-443.

[7] A. Viggiano, Combust. Flame 157 (2010) 328-340.

[8] S. Mukhopadhyay, J. Abraham, Combust. Flame 158 (2011) 1064 - 1075.

[9] S. Mukhopadhyay, J. Abraham, Combust. Flame 159 (2012) 2883 - 2895.

[10] S. Mukhopadhyay, J. Abraham, Proc. Inst. Mech. Eng. 227 (2012) 748-760.

[11] G. Borghesi, E. Mastorakos, R.S. Cant, Combust. Flame 160 (2013) $1254-1275$.

[12] A. Krisman, E.R. Hawkes, M. Talei, A. Bhagatwala, J.H. Chen, Proc. Combust. Inst. 35 (2015) 999-1006.

[13] S. Deng, P. Zhao, M.E. Mueller, C.K. Law, Combust. Flame 162 (2015) 3437-3445.

[14] S. Deng, P. Zhao, M.E. Mueller, C.K. Law, Combust. Flame 162 (2015) 4471 - 4478.

[15] E. Mastorakos, T.A. Baritaud, T.J. Poinsot, Combust. Flame 109 (1997) 198 - 223.

[16] E. Mastorakos, Prog. Energ. Combust. Sci. 35 (2009) 57-97. 
[17] R. Gordon, A. Masri, S. Pope, G. Goldin, Combust. Flame 151 (2007) 495-511.

[18] T.F. Lu, C.S. Yoo, J.H. Chen, C.K. Law, J. Fluid Mech. 652 (2010) 45-64.

[19] J. Chen, A. Choudhary, B. de Supinski, M. DeVries, E. Hawkes, S. Klasky, W. Liao, K. Ma, J. Mellor-Crummey, N. Podhorszki, R. Sankaran, S. Shende, C. Yoo, Comp. Sci. Disc. 2 (2009) 015001.

[20] A. Bhagatwala, Z. Luo, H. Shen, J.A. Sutton, T. Lu, J. Chen, Proc. Combust. Inst. 35 (2015) 1157-1166.

[21] J. Hinze, Turbulence, McGraw-Hill, New York, 1975.

[22] Y. Pei, E.R. Hawkes, M. Bolla, S. Kook, G.M. Goldin, Y. Yang, S.B. Pope, S. Som, Combust. Flame 168 (2016) $420-435$.

[23] P. Domingo, L. Vervisch, Proc. Combust. Inst. 26 (1996) 233 - 240.

[24] T. Echekki, J.H. Chen, Proc. Combust. Inst. 29 (2002) 2061-2068.

[25] R. Cabra, J.Y. Chen, R.W. Dibble, A.N. Karpetis, R.S. Barlow, Combust. Flame 143 (2005) 491 - 506.

[26] M.J. Papageorge, C. Arndt, F. Fuest, W. Meier, J.A. Sutton, Exp. Fluid. 55 (2014).

[27] H.G. Im, J.H. Chen, C.K. Law, Proc. Combust. Inst. 27 (1998) 1047 - 1056.

[28] T. Echekki, J.H. Chen, Combust. Flame 134 (2003) 169 - 191.

[29] X. Zheng, J. Yuan, C. Law, Proc. Combust. Inst. 30 (2005) 415 - 421.

[30] S. Cao, T. Echekki, Combust. Flame 151 (2007) 120 - 141.

[31] S.G. Kerkemeier, C.N. Markides, C.E. Frouzakis, K. Boulouchos, J. Fluid Mech. 720 (2013) 424-456.

[32] Y.B. Zel'dovich, Combust. Flame 39 (1980) $211-214$. 
[33] Y. Ju, C.B. Reuter, S.H. Won, Combust. Flame 162 (2015) 3580 - 3588.

[34] S. Singh, M.P.B. Musculus, R.D. Reitz, Combust. Flame 156 (2009) 1898 - 1908.

[35] A.J. Donkerbroek, A.P. van Vliet, L.M.T. Somers, N.J. Dam, J.J. ter Meulen, Combust. Flame $158(2011) 564-572$. 